\title{
Terahertz Detection by a Homodyne Field Effect Transistor Multiplicative Mixer
}

\author{
Sascha Preu, Sangwoo Kim, Ravi Verma, Peter G. Burke, Nguyen Q. Vinh, Mark S. Sherwin, and \\ Arthur C. Gossard, Life Fellow, IEEE
}

\begin{abstract}
We demonstrate an efficient scheme for mixing and down-conversion of two orthogonally polarized terahertz beams in a field effect transistor at frequencies far above frequencies where the transistor has gain. One signal is applied between gate and source and the other between drain and source. The mixer is a field detector with $960 \mathrm{pW} / \mathrm{Hz}$ noise-equivalent power at a local oscillator (LO) power of $8 \mu \mathrm{W}$. Orthogonal LO and signal power allows for simple diplexing.
\end{abstract}

Index Terms-Field-effect transistor (FET), mixer, terahertz (THz) detection.

\section{INTRODUCTION}

$\mathbf{S}$ EVERAL emerging applications for terahertz $(\mathrm{THz})$ imaging require $\mathrm{THz}$ detectors and imagers capable of detecting low levels of $\mathrm{THz}$ radiation. At cryogenic temperatures, hot electron bolometers [1] and transition edge bolometers [2] are capable of measuring single $\mathrm{THz}$ photons, but the cryogenic cooling requirement precludes most practical applications. At room temperature, Golay cells and pyroelectric detectors can detect powers above several $100 \mathrm{pW} / \sqrt{\mathrm{Hz}}$. These detectors, however, have too long time constants to enable fast $\mathrm{THz}$ imaging. In contrast, Schottky diode based room temperature $\mathrm{THz}$ detectors provide a fast response and can reach noise equivalent powers (NEPs) in the range of $4-30 \mathrm{pW} / \sqrt{\mathrm{Hz}}[3]$, [4].

Schottky diodes can also be used to mix two $\mathrm{THz}$ waves (frequency $\nu_{1}$ and $\left.\nu_{2}\right)$ to the intermediate frequency (IF) $\left(\nu_{\mathrm{IF}}=\right.$ $\left.\nu_{2}-\nu_{1}\right)$. The advantage of mixing is that a strong local oscillator

Manuscript received October 19, 2011; revised March 09, 2012; accepted March 10, 2012. Date of publication April 24, 2012; date of current version May 08, 2012. This work was supported by the Humboldt foundation, the NSF MRSEC program DMR-0520415 (MRL-UCSB), and the Air Force Office of Scientific Research, .via STTR Contract FA9550-10-C-0177.

S. Preu was with the Physics Department and Institute for Terahertz Science and Technology, University of California, Santa Barbara, CA 93106 USA , and also with the Materials Department, University of California, Santa Barbara, CA 93106 USA. He is now with the Chair of Applied Physics, University Erlangen-Nuremberg, 91058 Erlangen, Germany (e-mail: sascha.preu@ physik. uni-erlangen.de).

S. Kim is with the Tanner Research, Monrovia, CA 91016 USA.

R. Verma was with Tanner Research, Monrovia, CA 91016 USA. He is now with the Spectral Platforms Inc., La Canada, CA 91011 USA.

P. G. Burke and A. C. Gossard are with the Materials Department, University of California, Santa Barbara, CA 93106 USA.

N. Q. Vinh and M. S. Sherwin are with the Physics Department. and Institute for Terahertz Science and Technology, University of California, Santa Barbara, CA 93106 USA.

Color versions of one or more of the figures in this paper are available online at http://ieeexplore.ieee.org.

Digital Object Identifier 10.1109/TTHZ.2012.2191671
(LO) at one frequency is multiplied with a weak measurement (M) signal at a second frequency, thereby providing a stronger transducer input, and increasing the sensitivity. However, the mixing process in Schottky diodes is "additive" (the output is proportional to the square of the added E-fields $\left.\propto\left(E_{1}+E_{2}\right)^{2}\right)$. Only a small component of the transducer input is proportional to the multiplicative term $\left(\propto 2 E_{1} E_{2}\right)$, and a large component of the $\mathrm{LO}$ (and M) power is dissipated as heat $\left(\propto E_{1}^{2}+E_{2}^{2}\right)$. In particular, at high LO powers or signal powers, these additional signals generate currents, and therefore add noise compared to a device that is only sensitive to the mixing term. Recently, plasmonic $\mathrm{THz}$ field effect transistor (FET) detectors have gained much attention [5]-[8] as an alternative to Schottky detectors. A $\mathrm{THz}$ signal coupled between the drain (D) and source (S) self-rectifies by interaction of the two-dimensional electron gas (2DEG) in the channel with the gate (G) electrode. Direct detectors with room temperature NEPs in the range of $100 \mathrm{pW} / \sqrt{\mathrm{Hz}}$ have been demonstrated [8], [9] about 1-2 orders of magnitude worse than the best Schottky diodes.

These detectors can also be used in the additive "heterodyne" mode [10]. Glaab et al. [11] demonstrated a silicon FET heterodyne mixer with a NEP of $8 \mathrm{fW} / \mathrm{Hz}$ at a LO power of only $2 \mu \mathrm{W}$ and IF frequencies between $70 \mathrm{kHz}$ and $24 \mathrm{MHz}$. For additive mixing, measurement signal (M) and $\mathrm{LO}$ are fed to the same port. Some sort of coupler is required which is typically afflicted to losses.

Here, we demonstrate a multiplicative mixing architecture: a modulation-doped FET (MODFET) device with orthogonal gate-source and drain-source antennas, where the output is proportional to the product of two incident $\mathrm{THz}$ fields. In the mixing mode, the detector is sensitive to both the magnitude and the phase difference between the two mixed signals. An additional direct detection term is generated and may be read out depending on the operation mode, which is either $\sim P_{\mathrm{LO}}$ or $\sim P_{M}$ but not the sum of the two as in the case of additive mixing. This should reduce the noise caused by DC offsets due to direct detection signals.

The scheme of multiplicative mixing in FETs allows for homodyne mixing. In the three terminal FET, the LO signal and the measurement signal (M) are fed to two different ports namely the gate-source port and the drain-source port allowing for distinguishing LO from M, whereas in Schottky diodes, both signals have to be coupled to one port only. For homodyne detection in Schottky diodes, where $\omega_{\mathrm{LO}}=\omega_{\mathrm{M}}$, it is challenging to distinguish between $\mathrm{LO}$ and measurement signal. The FET geometry, where LO and M signals are orthogonal, also enables loss-free combination of LO and M with a wire 
grid polarizer. Furthermore, coupling of LO and signal to the FET can be optimized separately and independently, including antenna- and RF layout. We briefly review the theory of the mixing process which is elaborated in detail in [12]. Then we show experimental results on homodyne detection of two orthogonally polarized $\mathrm{THz}$ beams and compare the results to direct detection.

\section{THEORY}

The rectification in FET transistors originates from simultaneous modulation of the carrier velocity $v\left(U_{\mathrm{THz}}\right)$ and the carrier concentration, $n^{(2 D)}\left(U_{\mathrm{THz}}\right)$ by the $\mathrm{AC} \mathrm{THz}$ bias, $U_{\mathrm{THz}}(t)=$ $U_{\mathrm{THz}, 0} \cos (\omega t)$. The AC modulation of the current generates a DC component as $I \propto v\left(U_{\mathrm{THz}}\right) \cdot n^{(2 D)}\left(U_{\mathrm{THz}}\right) \propto U_{\mathrm{THz}}^{2}(t)$. The term $U_{\mathrm{THz}}^{2}(t)$ provides a DC component of $U_{\mathrm{THz}, 0}^{2} / 2$ [5], [12].

The room-temperature transistor detector used in this paper, is operated at a $\mathrm{THz}$ frequency, $\nu_{\mathrm{THz}}$, which is much smaller than the inverse momentum relaxation time of the carriers in the semiconductor, $\tau$, such that $\omega \tau \ll 1$. The $\mathrm{THz}$ fields in the gate region decay quickly, the plasmons are overdamped. No resonance occurs. The detector is thus operated in the nonresonant regime. In this limit, a $\mathrm{THz}$ bias coupled between the gate and source, $U_{g}^{\mathrm{THz}}$ and/or between the drain and source, $U_{\mathrm{DS}}^{\mathrm{THz}}$ creates a DC detector bias of [12]

$$
U_{r}=\eta\left[\frac{U_{g}^{\mathrm{THz}} U_{\mathrm{DS}}^{\mathrm{THz}} \cos \varphi}{2\left(U_{g}^{\mathrm{DC}}-U_{\mathrm{DS}}^{\mathrm{DC}}\right)}-\frac{\left(U_{\mathrm{DS}}^{\mathrm{THz}}\right)^{2}}{4\left(U_{g}^{\mathrm{DC}}-U_{\mathrm{DS}}^{\mathrm{DC}}\right)}\right]
$$

with $U_{g}^{\mathrm{DC}}=U_{G S}-U_{t h r}$ the $\mathrm{DC}$ bias at the gate below threshold, $U_{\mathrm{DS}}^{\mathrm{DC}}$ the DC drain-source bias, and $\varphi$ the phase between the DS and GS THz signals. The efficiency, $\eta$, includes losses at parasitic impedances, such as the access resistance. The second term in the brackets of (1) describes the direct detection. A single $\mathrm{THz}$ signal applied between the drain and source generates a DC response of the transistor, $U_{r} \propto\left(U_{\mathrm{DS}}^{\mathrm{THz}}\right)^{2}$. Coupling two THz signals between drain and source, $U_{\mathrm{DS}}^{\mathrm{THz}}(t)=U_{\mathrm{DS}}^{\mathrm{THz}}\left(\omega_{1} t\right)+U_{\mathrm{DS}}^{\mathrm{THz}}\left(\omega_{2} t\right)$, results in additive mixing by this term [10]. The first term in the brackets of (1), however, provides a multiplicative mixing response. The detection signal is proportional to the product of the gate-source AC voltage, $U_{g}^{\mathrm{THz}}$, and drain-source AC voltage, $U_{\mathrm{DS}}^{\mathrm{THz}}$. It is sensitive to the relative phase, $\varphi$, between $U_{g}^{\mathrm{THz}}$ and $U_{\mathrm{DS}}^{\mathrm{THz}}$. Note that (1) does not contain terms $\propto\left(U_{g}^{\mathrm{THz}}\right)^{2}$. No detection signal is expected from a single $\mathrm{THz}$ signal applied between the gate and source. This reduces heating and noise as compared to additive mixing where a term $\propto\left(U_{g}^{\mathrm{THz}}\right)^{2}$ is inevitable. However, we discussed in [12] that crosstalk within the device may lead to coupling of a GS signal to the channel under appropriate conditions, thus also providing a direct detection signal $\propto\left(U_{g}^{\mathrm{THz}}\right)^{2}$. By appropriate design and DC biasing of the transistor, the cross talk can be eliminated.

If two (ideal) antennas with radiation resistance $R_{A}$ are used to couple free space THz power to the FET, the response becomes

$$
U_{r}=\eta \frac{2 \sqrt{R_{A}^{G S} P_{\mathrm{THz}}^{G S}} \cdot \sqrt{R_{A}^{D S} P_{\mathrm{THz}}^{D S}} \cos \varphi-R_{A}^{D S} P_{\mathrm{THz}}^{D S}}{2\left(U_{g}^{\mathrm{DC}}-U_{\mathrm{DS}}^{\mathrm{DC}}\right)} .
$$

(a)

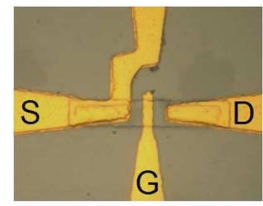

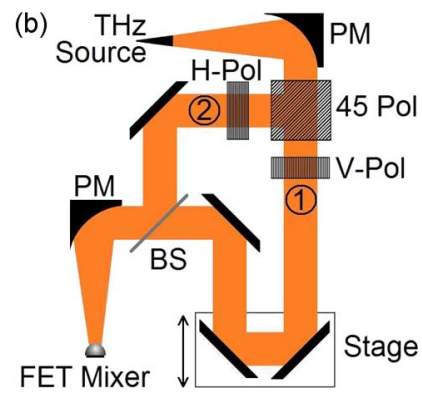

Fig. 1. (a) Micrograph of the sample layout. Two orthogonal bow-tie antennas are coupled to the device. Only the central part is shown. (b) Schematic of the experimental setup. The signal in arm1 couples to the DS antenna, the signal in arm 2 couples to the GS antenna.

The direct detection term is proportional to the THz power, whereas the mixing term is proportional the product of DS and GS THz fields, since $E^{i} \propto \sqrt{P_{\mathrm{THz}}^{i}}$.

\section{EXPERIMENTAL RESULTS}

In order to couple $\mathrm{THz}$ radiation independently between the gate-source and drain-source, we designed a structure with two orthogonal dipole-like antennas as illustrated in Fig. 1(a) and Fig. 2(c). The (Al)GaAs n-type depletion-mode MODFET layout is almost symmetric concerning the source and drain contacts and the gate position. This reduces nonlinear effects, such as asymmetries of the $I-V$ characteristics, or any undesired rectification processes, such as Schottky-effects by imperfect source or drain contacts as these effects cancel due to symmetry. The antenna that is coupled to source and drain responds to horizontal polarization, the antenna coupled to gate and source responds to vertical polarization. The upper arm of the gate-source antenna is shorted with one arm of the source-drain antenna which we arbitrarily define as the source side [c.f. Fig. 1(a)]. The other arm is connected to the gate.

The antenna-device system was simulated with the commercial finite element method electromagnetic simulation software COMSOL multiphysics. Fig. 2(a) shows the simulated power coupling performance compared to the measured device responsivity versus frequency. The experimental antenna resonance appears at $0.36 \mathrm{THz}$, close to the theoretically determined value of $0.325 \mathrm{THz}$. The radiation resistance at the resonance frequency of $0.325 \mathrm{THz}$ was determined to $540 \Omega$ [see Fig. 2(b)]. The cross talk (mututal coupling), i.e. the power of a signal with vertical polarization that couples to the antenna with horizontal alignment is shown in green in Fig. 2(a). A cross talk isolation of $20 \mathrm{~dB}$ is found at the theoretical resonance frequency.

Most measurements were performed between 0.308 and 0.42 $\mathrm{THz}$, matched to the resonance frequency of the device. The gate is about $1 \mu \mathrm{m}$ long and about $5.5 \mu \mathrm{m}$ wide. Due to these large dimensions, any rectification caused by the gate Schottky contact should be negligible. The remotely-doped channel is 57 $\mathrm{nm}$ below the gate. In order to reduce the crosstalk of the two signals within the device, a small reverse bias is required. At the optimum bias (around $-0.65 \mathrm{~V}$ ), no detector response is obtained for a GS signal when no DS signal is present, in agreement with (2). The transistor threshold bias is $U_{\mathrm{thr}} \approx-1.25 \mathrm{~V}$. The source and drain contacts behave ohmically but show a 


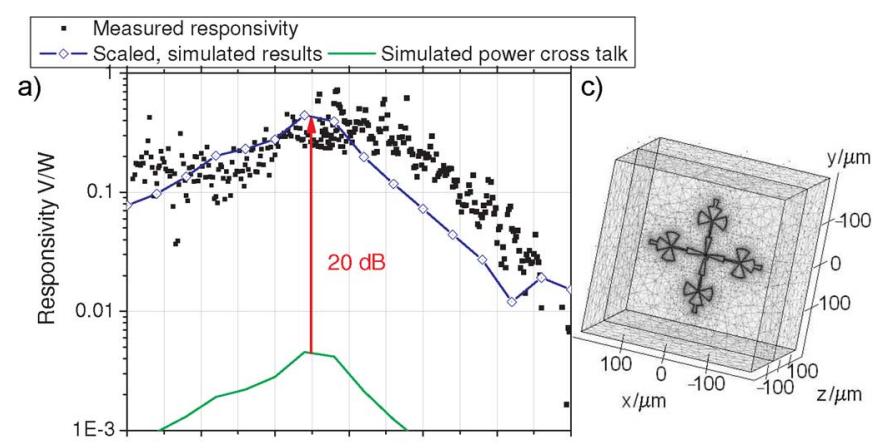

b)

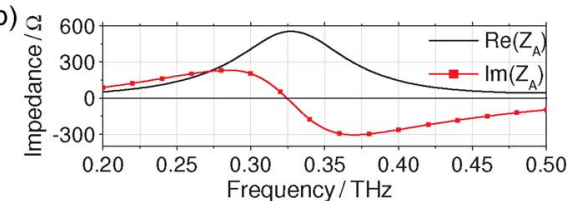

Fig. 2. (a) Measured responsivity of the device (black dots) compared to the simulated coupling efficiency of the $\mathrm{THz}$ power to the device (blue line with hollow squares). The simulated data are scaled to match the experimental data. The simulated cross talk is shown in green. (b) Simulated real and imaginary part of the impedance of the antenna-filter system. (c) Simulation area. The cloverleave like filter structures terminate the antenna arms.

source-drain (DC) resistance of 9.3 and $18 \mathrm{k} \Omega$ at $U_{\mathrm{GS}}=0 \mathrm{~V}$ and $U_{\mathrm{GS}}=-0.65 \mathrm{~V}$, reflecting large access and contact resistances. The channel resistance is calculated to be $1.8 \mathrm{k} \Omega$ at $U_{\mathrm{GS}}=0 \mathrm{~V}$. We estimate an upper limit for the RC cut-off frequency $f_{\mathrm{RC}}<20 \mathrm{GHz}$ [12]. An amplifier (SR560) with an amplification factor of 10 or 100 and a high-pass filter, blocking frequencies below $10 \mathrm{~Hz}$ is used prior to lock-in detection.

The experimental setup is shown in Fig. 1(b). The horizontally polarized $\mathrm{THz}$ beam generated by a frequency-multiplied microwave oscillator is equally split by a $45^{\circ}$ oriented wire grid polarizer. Subsequent vertical (V-Pol) and horizontal (H-Pol) wire grids provide a horizontally polarized beam in arm1 aligned with the source-drain antenna of the device and a vertically polarized beam in arm 2 for exciting the source-gate antenna. A delay stage is included in arm1. The two beams are combined by a non-polarizing beam splitter or a vertical wire grid (BS) and focused onto the sample by a parabolic mirror (PM). A hyper-hemispheric, high-resistivity silicon lens is used to enhance the coupling of the free space radiation to the device. A mechanical chopper for lock-in detection is introduced in either arm1 or arm2 or directly in front of the source.

As the beams in arm 1 and arm 2 are orthogonally polarized, no interference fringes should appear by tuning the stage in arm1, in contrast to a Michelson interferometer. This was confirmed with a polarization-insensitive detector. Only small oscillations were found, attributed to reflections and stray light of the highly coherent $\mathrm{THz}$ signal. The orthogonal polarization in the two arms allows for practically loss-free combination of the two signals by a polarizer (wire grid).

In order to be sensitive only to the mixing part described by the first term in (2), we chop $\left(\nu_{\mathrm{ch}}=50 \mathrm{~Hz}\right)$ the GS signal (arm2) only. This way, only $U_{g}^{\mathrm{THz}}$ is modulated (mixing mode), the direct detection response according to (2) remains unmodulated and is not detected by the lock-in. The power in the GS arm was attenuated by adding two further wire grids in the path as
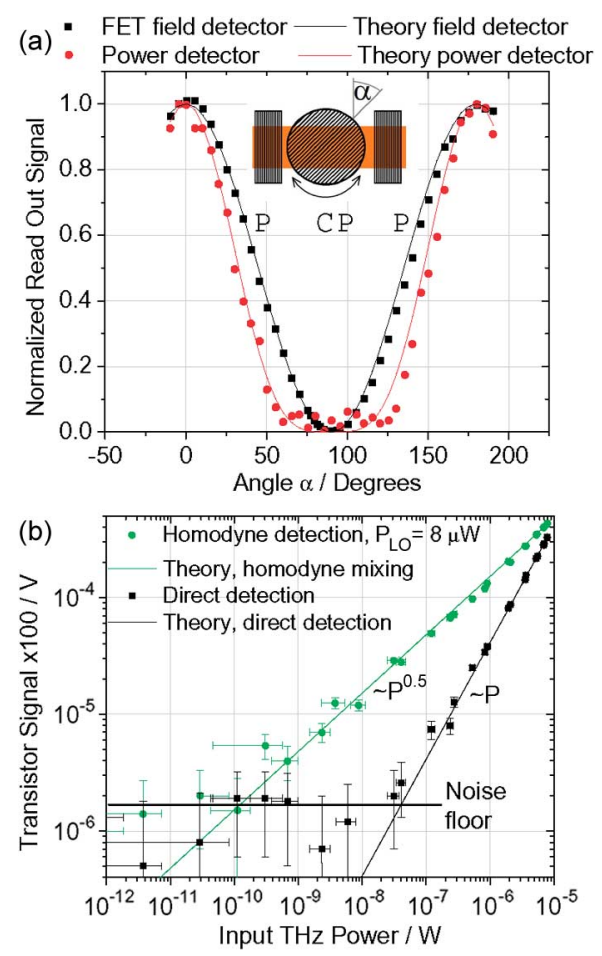

Fig. 3. (a) Normalized detected signal at $0.310 \mathrm{THz}$ for chopped GS power $(\operatorname{arm} 2)$ versus angle of the center polarizer $(\mathrm{CP})$ relative to outer wire grids $(\mathrm{P})$ as shown in the inset. Black squares: measured field detection response of the FET for varying the GS power. Red circles: Response of a power-law detector (Thomas Keating photo-acoustic power meter). Theoretical curves are included for both cases. (b) FET detection signal at $0.373 \mathrm{THz}$ versus $\mathrm{THz}$ power. Green circles: Mixing mode signal (GS power chopped). The detection signal is proportional to the square root of the power fed to the GS antenna. The LO power (DS signal) is $8 \mu \mathrm{W}$, limited by the setup. Black squares: Direct detection signal: the DS signal (arm1) is chopped, the GS (arm2) is blocked. The FET detector shows a linear power response.

illustrated in the inset of Fig. 3(a). Fig. 3(a) clearly shows the $\cos ^{2} \alpha$-dependence of a field detector (black squares). As the mixing term in (2) depends on the product of $U_{g}^{\mathrm{THz}}$ and $U_{\mathrm{DS}}^{\mathrm{THz}}$, a similar curve is obtained if the power in $\operatorname{arm} 1\left(\propto\left(U_{\mathrm{DS}}^{\mathrm{THz}}\right)^{2}\right)$ is altered, although this beam is not modulated. In particular, the detected signal vanishes by blocking the power in either DS- or GS arm as expected from (2). In this "mixing" mode, the FET mixes a (strong) local oscillator (LO) DS signal with the GS signal. As a control, we repeated the measurement with a polarization-insensitive Thomas Keating detector. This shows the expected $\cos ^{4} \alpha$ dependence of a power detector when varying the GS power, as illustrated in Fig. 3(a), circles. The setup can also be used to drive the FET as direct power detector by blocking the GS signal and chopping the DS signal, i.e. modulating $U_{\mathrm{DS}}^{\mathrm{THz}}$ only (direct detection mode). In that case, the transistor provides a read-out signal as $\cos ^{4} \alpha$ for the same attenuation setup in the DS arm (arm1) as expected for a power detector.

Similar measurements of both the mixing term and direct detection at $0.373 \mathrm{THz}$ versus power are shown in Fig. 3(b). In the mixing mode, the detector response is $\propto \sqrt{P_{\mathrm{GS}}}$, there is no linear contribution $\propto P_{\mathrm{GS}}$. This clearly shows that the cross talk of GS power to the channel was eliminated by an appropriate device structure and DC gate bias. Using the maximum available LO power of $8 \mu \mathrm{W}$, a noise floor of $120 \mathrm{pW}$ at $0.125 \mathrm{~Hz}$ lock-in bandwidth is achieved in the mixing mode. The direct 
detection signal is proportional to the DS power, as expected from the direct detection term in (2). The noise floor for direct detection is $44 \mathrm{nW}, 360$ times higher than the mixing detection at these settings. From the values presented above, the mixing $\mathrm{NEP}$ is about $0.96 \mathrm{nW} / \mathrm{Hz}$ and the direct detection NEP is about $130 \mathrm{nW} / \sqrt{\mathrm{Hz}}$. This relatively large noise floor is due to the lack of impedance matching of antenna and sample and the relatively high (DC) resistance of the sample of $18 \mathrm{k} \Omega$, mainly caused by a high access resistance. This causes a thermal noise amplitude of an estimated $U_{N} / \Re=42 \mathrm{nW} / \sqrt{\mathrm{Hz}}$ for direct detection and $0.11 \mathrm{nW} / \mathrm{Hz}$ for mixing with $P_{\mathrm{LO}}=8 \mu \mathrm{W}$ for this detector, where $U_{N}$ is the thermal voltage noise and $\Re \approx 0.4 \mathrm{~V} / \mathrm{W}$ is the responsivity of the detector. The theoretical value for direct detection is only a factor 3 smaller than the measured value of 130 $\mathrm{nW} / \sqrt{\mathrm{Hz}}$. Additional noise could have originated from drifts of the gate bias of the device and stray noise received by the detector from other noise sources, such as power supplies and such alike. The mixing noise scales as the square of the direct detection noise. A factor of 3 higher noise floor for direct detection results in a factor of $3^{2}=9$ higher noise floor for mixing, providing an NEP $=9 \times 0.11 \mathrm{nW} / \mathrm{Hz}=0.99 \mathrm{nW} / \mathrm{Hz}$. This is very close to the experimental value of $0.96 \mathrm{nW} / \mathrm{Hz}$. The noise floors for direct detection and mixing are in good agreement.

This first generation device had not been optimized for impedance matching and shows a very high access resistance. For an ideal device, the coupling efficiency can theoretically reach $(1-30 \%) \times 50 \%=35 \%$, since $30 \%$ of the power is reflected by the silicon lens and $50 \%$ of the power is transmitted to the load for the impedance matched case. The simulated coupling efficiency for a device resistance of $10 \mathrm{k} \Omega$, similar to the experimental value, is only $5.6 \%$ due to impedance mismatch. The direct detection results shown here can be improved by a factor of 6.25 simply by lowering the device resistance for impedance matching of antenna and device. A lower device resistance also results in lower thermal noise since the (direct detection) noise power is proportional to $\sqrt{R}$. For the impedance matched case of $R=R_{A}=540 \Omega$, the direct detection noise would be lowered by a factor of 4.4 , providing a total improvement of the NEP by a factor of $6.25 \times 4.4=28$ for direct detection, and a factor of 750 for mixing. A substantial part of the RF power is also lost at the relatively large access resistance of the device. For further improvement of the NEP, the intrinsic responsivity of the detector element can be significantly improved by careful optimization of the device parameters, such as larger channel doping, smaller channel depth and optimized device geometry [12]. As the device architecture is -on principle- similar to other antenna coupled FET rectifiers and mixers, an optimized multiplicative mixer should be able to provide similar NEPs than those mentioned in the literature [11]. With the design presented in this paper, however, LO and $\mathrm{M}$ can be combined loss-free improving the device performance. The values presented in the literature did not yet reach any theoretical limit. In [12], we estimate ideal direct detection noise floors in the sub $-\mathrm{pW} / \sqrt{\mathrm{Hz}}$ and mixing noise floors in the sub-aW/Hz range for $\mathrm{LO}$ powers around $1 \mu \mathrm{W}$, however, assuming that thermal noise is the dominant noise term. Thus, FET mixers can be competitive to state-of-the-art Schottky diodes.

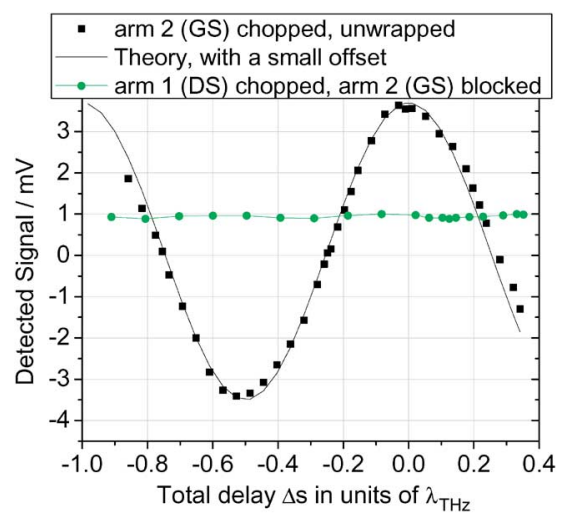

Fig. 4. Deconvoluted bias generated by the FET detector for chopped GS signal versus delay of the DS signal. Except a small offset, the response agrees well with the $\cos \varphi$-dependence described by term 1 in (2). To exclude artifacts, the green circles show the same measurement with the DS beam chopped and the GS beam blocked (direct detection mode of the DS signal).

A mixer allows for measuring both field amplitude, as shown in Fig. 3(b), and phase, as illustrated in Fig. 4. To verify the phase sensitive detection, the delay, $\Delta s$, of the DS arm was varied by the stage in arm1, while the GS signal in arm2 was chopped (mixing mode). Taking both the lock-in phase and amplitude into account, we can reconstruct the bias generated by the rectification process. The phase, $\varphi=2 \pi \Delta s / \lambda$, is introduced between DS (arm1) and GS (arm2) by tuning the delay stage. The read out signal was found to be proportional to $\cos \varphi$, as expected from (2). To exclude artifacts, the measurement was repeated with a photo-acoustic power law detector and with the FET in the direct detection mode, where the FET only receives the signal in the delayed arm. In both cases, only a slight oscillation of the detected signal was found, attributed to reflections of the highly coherent CW signal within the setup.

Phase-sensitive detection, as illustrated in Fig. 4, is important for (continuous-wave) distance determination and 3D image reconstruction [13], as the phase provides depth information. Orthogonally polarized local oscillator and measurement signals, as shown here, allow for simple diplexing of both beams, for instance with a wire grid.

The FET can also be operated in a heterodyne detection mode by feeding signals with a slightly different intermediate frequency (IF), $\Delta \nu_{\mathrm{IF}}$, to source-drain $\left(\nu_{\mathrm{THz}}\right)$ and gate-source $\left(\nu_{\mathrm{THz}}+\Delta \nu_{\mathrm{TF}}\right)$, resulting in a time-dependent phase $\varphi(t)=2 \pi \Delta \nu_{\mathrm{IF}} t$. A large value for $\Delta \nu_{\mathrm{IF}}$ suppresses contributions from $1 / f$-noise of the FET and amplification circuit.

\section{CONCLUSION}

In conclusion, we presented experimental results for multiplicative mixing at $\mathrm{THz}$ frequencies in a MODFET. We demonstrated mixing of two orthogonally polarized $\mathrm{THz}$ beams in a homodyne detection mode by coupling the signals to an orthogonal set of drain-source and gate-source antennas. Despite a fairly high device impedance and no impedance matching to the antenna, we achieved $960 \mathrm{pW} / \mathrm{Hz}$ NEP. The mixer detects both field amplitude and relative phase between the two beams. Improvement of the design, such as impedance matching of the antenna to the device and a reduction of losses caused by a 
large access resistance should allow the reduction of the noise floor and strongly improve the responsivity to values competitive with Schottky diodes. As LO signal and measurement signal are orthogonally polarized, loss-free combination of LO and measurement beams by a wire grid was demonstrated.

\section{REFERENCES}

[1] B. Karasik, D. Olaya, J. Wei, S. Pereverzev, M. Gershenson, J. Kawamura, W. McGrath, and A. Sergeev, "Record-low NEP in hot-electron titanium nanobolometers," IEEE Trans. Appl. Supercond., vol. 17, no. 2, pt. 1, pp. 293-297, Jun. 2007.

[2] A. Luukanen and J. P. Pekola, "A superconducting antenna-coupled hot-spot microbolometer," Appl. Phys. Lett., vol. 82, pp. 3970-3972, 2003.

[3] C. Sydlo, O. Cojocari, D. Schönherr, T. Goebel, P. Meissner, and H. Hartnagel, "Fast THz detectors based on InGaAs Schottky diodes," Frequenz, vol. 62, no. 5-6, pp. 107-110, 2008.

[4] E. Brown, A. C. Young, J. D. Z. J. E. Bjarnason, and A. C. Gossard, "Millimeter and sub-millimeter wave performance of an eras: InGaAs Schottky diode coupled to a single turn square spiral," Int. J. High Speed Electron., vol. 17, no. 2, pp. 383-394, 2007.

[5] D. Veksler, F. Teppe, A. P. Dmitriev, V. Y. Kachorovskii, W. Knap, and M. S. Shur, "Detection of terahertz radiation in gated two-dimensional structures governed by dc current," Phys. Rev. B, vol. 73, p. 125328, 2006.

[6] D. Coquillat, S. Nadar, F. Teppe, N. Dyakonova, S. Boubanga-Tombet, W. Knap, T. Nishimura, T. Otsuji, Y. M. Meziani, G. M. Tsymbalov, and V. V. Popov, "Room temperature detection of sub-terahertz radiation in double-grating-gate transistors," Opt. Express, vol. 18, no. 6, pp. 6024-6032, 2010

[7] G. C. Dyer, S. Preu, G. R. Aizin, J. Mikalopas, A. D. Grine, J. L. Reno, J. M. Hensley, N. Q. Vinh, A. C. Gossard, M. S. Sherwin, S. J. Allen, and E. A. Shaner, "Enhanced performance of resonant sub-terahertz detection in a plasmonic cavity," Appl. Phys. Lett., vol. 100, p. 083506, 2012.

[8] A. Lisauskas, U. Pfeiffer, E. Ojefors, P. H. Bolivar, D. Glaab, and H. G. Roskos, "Rational design of high-responsivity detectors of terahertz radiation based on distributed self-mixing in silicon field-effect transistors," J. Appl. Phys., vol. 105, no. 11, p. 114511, 2009.

[9] R. Tauk, F. Teppe, S. Boubanga, D. Coquillat, W. Knap, Y. M. Meziani, C. Gallon, F. Boeuf, T. Skotnicki, C. Fenouillet-Beranger, D. K. Maude, S. Rumyantsev, and M. S. Shur, "Plasma wave detection of terahertz radiation by silicon field effects transistors: Responsivity and noise equivalent power," Appl. Phys. Lett., vol. 89, no. 25, p. 253511, 2006.

[10] B. Gershgorin, V. Kachorovskii, Y. Lvov, and M. Shur, "Field effect transistor as heterodyne terahertz detector," Electron. Lett., vol. 44, no. 17, pp. 1036-1037, 2008.

[11] D. Glaab, S. Boppel, A. Lisauskas, U. Pfeiffer, E. Ojefors, and H. G. Roskos, "Terahertz heterodyne detection with silicon field-effect transistors," Appl. Phys. Lett., vol. 96, no. 4, p. 042106, 2010.

[12] S. Preu, S. Kim, R. Verma, P. G. Burke, M. S. Sherwin, and A. C. Gossard, "An improved model for non-resonant terahertz detection in field effect transistors," J. Appl. Phys., vol. 111, no. 2, p. 024502, 2012.

[13] K. J. Siebert, H. Quast, R. Leonhardt, T. Löffler, M. Thomson, T. Bauer, H. G. Roskos, and S. Czasch, "Continuous-wave all-optoelectronic terahertz imaging," Appl. Phys. Lett., vol. 80, no. 16, pp. 3003-3005, 2002.

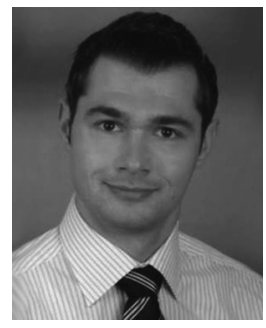

Sascha Preu received the Diploma degree and the Ph.D. degree in physics (summa cum laude) from the Univ. of Erlangen-Nuremberg, Erlangen, Germany, in 2005 and 2009, respectively.

From 2004 to 2010, he was with the Max Planck Institute for the Science of Light, Erlangen, Germany. During 2010-2011, he worked at the Materials and Physics Department, University of Santa Barbara, CA. Currently, he is at the Chair of Applied Physics, University Erlangen-Nuremberg, Erlangen, Germany. His research interests focus on the development of semiconductor-based $\mathrm{THz}$ sources and detectors. He also works on applications of $\mathrm{THz}$ radiation, in particular the characterization of novel $\mathrm{THz}$ optics and materials such as whispering gallery mode resonators and graphene.

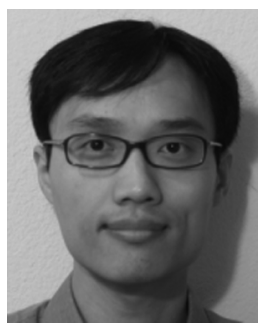

Sangwoo Kim received the B.S. degree in physics from Korea University, Seoul, South Korea, in 2000, and the M.S. and Ph.D. degrees in physics from the University of California, Santa Barbara, in 2005 and 2009 , respectively.

$\mathrm{He}$ is a member of the Staff Scientists at Tanner Research, Inc., Monrovia, CA, since 2009, where he is involved in the development of plasmonic infrared detectors and terahertz mixers.

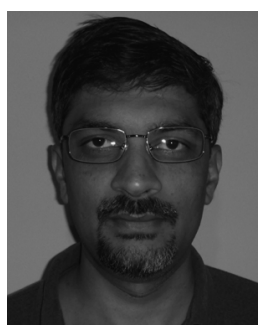

Ravi Verma received the B.Tech. degree in metallurgical engineering from Indian Institute of Technology (IIT), Kharagpur, India, in 1990, and the Ph.D. degree in materials science from Virginia Tech, Blacksburg, in 1994.

From 1994 to 1996, he was a Post-Doctoral scholar at Virginia Tech with extensive commitments at DuPont Central Research \& Development. From 1996 to 1999, he was a Post-Doctoral Scholar at the California Institute of Technology (Caltech), Pasadena. He was employed as a Senior Scientist at Tanner Research from May 2001 to May 2011. Since June 2011, he is the $\mathrm{CEO}$ and founder of Spectral Platforms, which is developing rapid diagnostics tools for infectious diseases. He is currently with Spectral Platforms Inc., La Canada, CA.

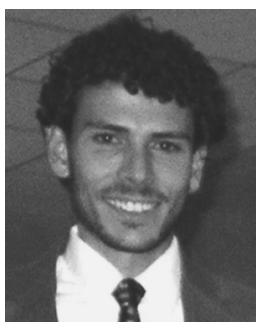

Peter G. Burke received the B.S. degree in Materials Science and Engineering at Lehigh University, Philadelphia, PA, in 2008. He is currently working toward the Ph.D. degree in Materials Science Department, University of California, Santa Barbara, under the guidance of A. C. Gossard and J. E. Bowers.

His research covers the growth (by molecular beam epitaxy), characterization, and electronic measurement of thin film III-V semiconductors for thermoelectric applications.

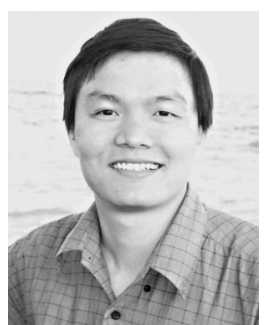

Nguyen Quang Vinh received the M.Sc. degree from the Institute of Physics, Hanoi, Vietnam, in 1999 and the $\mathrm{Ph} . \mathrm{D}$. degree in physics from the University of Amsterdam, Amsterdam, The Netherlands, in 2004.

$\mathrm{He}$ is presently with the Institute for Terahertz Science and Technology, Department of Physics, University of California, Santa Barbara. Previously, he was a Senior United Kingdom Facility Scientist with the Dutch Free-Electron Laser for Infrared Experiment (FELIX), FOM Institute for Plasma Physics Rijnhuizen, The Netherlands. His research interests include the optoelectronic materials for optical interconnect; terahertz coherent control for quantum information processing; ultra-fast dynamics of intersubband transitions in quantum systems; dynamics of hydration shell and biomolecules/ions in liquid water by the terahertz spectroscopy for its biological functions. 


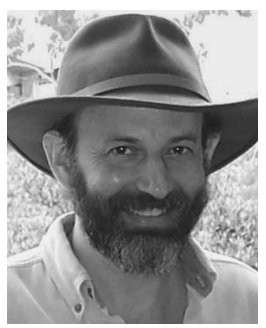

Mark Sherwin received the Ph.D. degree in experimental solid state physics from the University of California (UC), Berkeley, in 1988.

He then joined the faculty of the Physics Department at UC Santa Barbara. He is the Director of the Institute for Terahertz Science and Technology at UC Santa Barbara, the home of the UC Santa Barbara Free-Electron Lasers. His current research interests include terahertz detectors, the nonlinear response of semiconductor heterostructures to intense terahertz radiation, high-field pulsed electron paramagnetic resonance at $240 \mathrm{GHz}$, and the terahertz dynamics of water.

Dr. Sherwin is a Fellow of the American Physical Society.

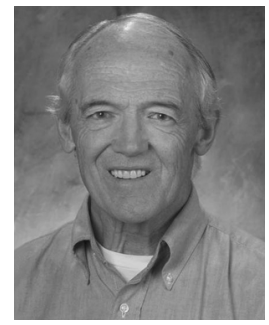

Arthur Gossard (SM'88-F'01-LF'12) is Professor of Materials and Electrical and Computer Engineering at University of California, Santa Barbara. His special interests are molecular beam epitaxy, the growth of quantum wells and superlattices, and their applications to high performance electrical and optical devices.

Dr. Gossard is a fellow of the American Physical Society, AAAS, and a member of the National Academy of Engineering, and the National Academy of Sciences. 